\title{
CONVECTIVE OVERSTABILITY IN ACCRETION DISKS: THREE-DIMENSIONAL LINEAR ANALYSIS AND NONLINEAR SATURATION
}

\author{
WLADIMIR LYRA ${ }^{1,2,3}$ \\ ${ }^{1}$ Jet Propulsion Laboratory, California Institute of Technology, 4800 Oak Grove Drive, Pasadena, CA 91109, USA; wlyra@ caltech.edu \\ 2 Department of Geology and Planetary Sciences, California Institute of Technology, 1200 E. California Avenue, Pasadena, CA 91125, USA \\ Received 2014 April 21; accepted 2014 May 13; published 2014 June 17
}

\begin{abstract}
Recently, Klahr \& Hubbard claimed that a hydrodynamical linear overstability exists in protoplanetary disks, powered by buoyancy in the presence of thermal relaxation. We analyze this claim, confirming it through rigorous compressible linear analysis. We model the system numerically, reproducing the linear growth rate for all cases studied. We also study the saturated properties of the overstability in the shearing box, finding that the saturated state produces finite amplitude fluctuations strong enough to trigger the subcritical baroclinic instability (SBI). Saturation leads to a fast burst of enstrophy in the box, and a large-scale vortex develops in the course of the next $\approx 100$ orbits. The amount of angular momentum transport achieved is of the order of $\alpha \approx 10^{-3}$, as in compressible SBI models. For the first time, a self-sustained three-dimensional vortex is produced from linear amplitude perturbation of a quiescent base state.
\end{abstract}

Key words: hydrodynamics - instabilities - methods: analytical - methods: numerical - planets and satellites: formation - protoplanetary disks

Online-only material: color figures

\section{INTRODUCTION}

Accretion in disks is generally thought to occur by the action of turbulence, for which magnetorotational instability (MRI; Balbus \& Hawley 1991) is the most likely culprit. However, protoplanetary disks are cold; the ionization level required to couple the gas to the ambient field is not always met (Blaes \& Balbus 1994), leading to zones that are "dead" to the MRI (Gammie 1996; Turner \& Drake 2009). So, the quest for hydrodynamical sources of turbulence continues, if only to provide accretion through this dead zone.

One such possible source of hydrodynamical turbulence is the subcritical baroclinic instability (SBI; Klahr \& Bodenheimer 2003; Klahr 2004; Petersen et al. 2007a, 2007b; Lesur \& Papaloizou 2010; Lyra \& Klahr 2011; Raettig et al. 2013), a process shown to sustain large-scale vortices in the presence of a radial entropy gradient and thermal relaxation or diffusion. Twodimensional linear stability analysis and numerical simulations do not find instability if only seeded with linear noise (Johnson \& Gammie 2005), though it was shown that finite amplitude perturbations would trigger it, concluding that the instability is nonlinear in nature (Lesur \& Papaloizou 2010). Characterization of the instability through nonlinear numerical simulations shows that maximum amplification is found for thermal times in the range of 1-10 times the dynamical timescale (Lesur \& Papaloizou 2010; Lyra \& Klahr 2011; Raettig et al. 2013). Although no criterion for a critical Reynolds number was derived, Raettig et al. (2013) show that as resolution is increased, ever smaller perturbations are necessary, as expected if the process is physical. Compressible simulations (Lesur \& Papaloizou 2010; Lyra \& Klahr 2011) show that the spiral density waves excited by the vortices (Heinemann \& Papaloizou 2009a, 2009b, 2012) transport angular momentum at the level of $\alpha \approx 10^{-3}$, where $\alpha$ is the Shakura-Sunyaev parameter (Shakura \& Sunyaev 1973). If this process indeed occurs in disks, it would provide not only

\footnotetext{
3 Sagan Fellow.
}

accretion but also a fast route for planet formation in the dead zone, since vortices speed up the process enormously by concentrating particles in their centers (Barge \& Sommeria 1995; Klahr \& Bodenheimer 2006; Lyra et al. 2008b, 2009).

The appeal of the SBI, however, is severely hindered by its nonlinear nature. Without the guidance of analytics, nonlinear processes are difficult to characterize, and the accuracy of the numerics has to be well-established beyond reasonable doubt. Recently, Klahr \& Hubbard (2014, hereafter KH14) have claimed that, when considering the same equations that lead to SBI in two dimensions, linear growth exists if vertical wavelengths are considered. The unstable mode is a slowly growing epicyclic oscillation, which led the authors to name the process "convective overstability." Growth is powered by buoyancy and thermal relaxation in the same regime as the SBI, with cooling time of the order of the dynamical time. We analyze this claim of linearity in more detail in this paper. Independent verification is desirable since unorthodox assumptions were made in the linear analysis in KH14. In particular, the authors assumed that the timescale for pressure equilibration is fast, and thus set the pressure perturbation to zero in the linear analysis. Because of this strong assumption, skepticism about the validity of the work naturally remains until a rigorous derivation of the dispersion relation is provided, unambiguously demonstrating that the eigenvector of the growing root has no appreciable pressure term. In this work, we provide such derivation.

Another point raised by KH14 is the connection between this overstability and the SBI, if any. A priori, the two processes have little to do with each other. However, as the regimes of cooling time for both are similar, if convective overstability exists, it may generate finite amplitude perturbations that trigger the SBI. In this scenario, the (nonlinear) SBI would simply be the saturated state of the (linear) convective overstability. Since the difficulty of finding a source of finite amplitude perturbation in dead zones in the required range of cooling times made the SBI look less attractive as a relevant disk process, a linear process that can spawn the SBI from arbitrarily low-level noise would be 
particularly interesting. Conversely, there is the possibility, of course, that the saturated state of the convective overstability may still be of too low amplitude to trigger the SBI. We investigate these possibilities in the present study.

This paper is structured as follows. In Section 2, we perform a linear analysis calculating the full compressible dispersion relation. In Section 3, we take the anelastic limit to derive the instability criterion, finding the roots, the most unstable mode, and associated eigenvector. In Section 4, we perform numerical simulations in the shearing box to characterize the linear growth phase and nonlinear saturation in two dimensions and three dimensions. We conclude in Section 5.

\section{LINEAR DISPERSION RELATION}

Let us consider the compressible Euler equations with thermal relaxation.

$$
\begin{aligned}
\frac{\partial \rho}{\partial t}+(\boldsymbol{u} \cdot \nabla) \rho & =-\rho \boldsymbol{\nabla} \cdot \boldsymbol{u}, \\
\frac{\partial \boldsymbol{u}}{\partial t}+(\boldsymbol{u} \cdot \nabla) \boldsymbol{u} & =-\frac{1}{\rho} \nabla p+\boldsymbol{g}, \\
\frac{\partial p}{\partial t}+(\boldsymbol{u} \cdot \nabla) p & =-\gamma p \nabla \cdot \boldsymbol{u}-\frac{p}{T} \frac{\left(T-T_{0}\right)}{\tau},
\end{aligned}
$$

where $\rho$ is the density, $\boldsymbol{u}$ is the velocity, $p$ is the pressure, $\gamma$ is the adiabatic index, $T$ is the temperature, $T_{0}$ is a reference temperature, and $\tau$ is the thermal time. We consider the cylindrical approximation, meaning that we omit the vertical component of the stellar gravity, as well as vertical stratification. In this approximation, the gravity is $\boldsymbol{g}=-\Omega^{2} \boldsymbol{r}$, with $\Omega$ the Keplerian angular frequency and $\boldsymbol{r}$ the cylindrical radial coordinate. A list of the mathematical symbols used in this work, together with their definitions, is provided in Table 1.

We linearize Equations (1)-(3) into the base state and perturbation (the latter denoted by primes) as $u_{r}=u_{r}^{\prime}, u_{\phi}=u_{\phi}^{\prime}+\Omega r$, $u_{z}=u_{z}^{\prime}, p=p_{0}+p^{\prime}$, and $\rho=\rho_{0}+\rho^{\prime}$. Assuming cylindrical approximation ( $\partial_{z}=0$ for the base state), Equations (1)-(3) become

$$
\begin{aligned}
\partial_{\hat{t}} \rho^{\prime}+u_{r}^{\prime} \partial_{r} \rho_{0}+\rho_{0} \nabla \cdot \boldsymbol{u}^{\prime} & =0, \\
\partial_{\hat{t}} u_{r}^{\prime}-2 \Omega u_{\phi}^{\prime}+\frac{1}{\rho_{0}} \partial_{r} p^{\prime}-\frac{\rho^{\prime}}{\rho_{0}^{2}} \partial_{r} p_{0} & =0, \\
\partial_{\hat{t}} u_{\phi}^{\prime}+\Omega(2-q) u_{r}^{\prime}+\frac{1}{\rho_{0}} \partial_{\hat{\phi}} p^{\prime} & =0, \\
\partial_{\hat{t}} u_{z}^{\prime}+\frac{1}{\rho_{0}} \partial_{z} p^{\prime} & =0, \\
\partial_{\hat{t}} p^{\prime}+u_{r}^{\prime} \partial_{r} p_{0}+\gamma p_{0} \nabla \cdot \boldsymbol{u}^{\prime}+\frac{p^{\prime}}{\tau}-\frac{p_{0} \rho^{\prime}}{\rho_{0} \tau} & =0,
\end{aligned}
$$

\begin{tabular}{|c|c|c|}
\hline 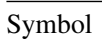 & Definition & Description \\
\hline$r$ & & Cylindrical radial coordinate \\
\hline$\phi$ & & Azimuth \\
\hline$r_{0}$ & & Reference radius \\
\hline$x$ & $=r-r_{0}$ & Cartesian radial coordinate \\
\hline$y$ & $=r \phi$ & Cartesian azimuthal coordinate \\
\hline$z$ & & Vertical coordinate \\
\hline$k_{r}, k_{x}$ & & Radial wavenumber \\
\hline$m$ & & Azimuthal wavenumber \\
\hline$k_{z}$ & & Vertical wavenumber \\
\hline$k$ & $=\sqrt{k_{r}^{2}+k_{z}^{2}}$ & \\
\hline$\mu$ & $=k_{z} / k$ & \\
\hline$t$ & & Time \\
\hline$\rho$ & & Density \\
\hline$u$ & & Velocity \\
\hline$T$ & & Temperature \\
\hline$\gamma$ & & Adiabatic index \\
\hline$c_{p}$ & & Specific heat at constant pressure \\
\hline$c_{v}$ & $=c_{p} / \gamma$ & Specific heat at constant volume \\
\hline$p$ & $=c_{v}(\gamma-1) \rho T$ & Pressure \\
\hline$\tau$ & & Thermal time \\
\hline 5 & $=1 / \gamma \tau$ & \\
\hline$\Omega$ & & Keplerian angular frequency \\
\hline$q$ & $=-d \ln \Omega / d \ln r$ & Shear parameter \\
\hline$\kappa$ & $=\sqrt{2(2-q)} \Omega$ & Epicyclic frequency \\
\hline$\alpha$ & $=d \ln \rho / d \ln r$ & Density gradient \\
\hline$\beta$ & $=d \ln T / d \ln r$ & Temperature gradient \\
\hline$\omega$ & & Complex eigenfrequency \\
\hline $\bar{\omega}$ & $=\omega-m \Omega$ & \\
\hline$s$ & $=\operatorname{Re}(\bar{\omega})$ & Oscillation frequency \\
\hline$\sigma$ & $=\operatorname{Im}(\bar{\omega})$ & Growth rate \\
\hline$c$ & $=\left[\begin{array}{ll}T & c_{p}(\gamma-1)\end{array}\right]^{1 / 2}$ & Sound speed \\
\hline$A$ & $=c \partial_{r} \ln \rho$ & \\
\hline$B$ & $=\gamma^{-1} c \partial_{r} \ln p$ & \\
\hline$N$ & $=\sqrt{A B-B^{2}}$ & Brunt-Väisälä frequency \\
\hline$a$ & $=A / c$ & \\
\hline$b$ & $=B / c$ & \\
\hline$H$ & $=c / \Omega$ & Disk scale height \\
\hline$h$ & $=H / r$ & Disk aspect ratio \\
\hline$\xi$ & $=\alpha+\beta$ & Pressure gradient \\
\hline$\Sigma$ & $=\propto \rho H$ & Surface density \\
\hline$\psi$ & $=d \ln \Sigma / d \ln r$ & Surface density gradient \\
\hline
\end{tabular}

where $\partial_{\hat{t}}=\partial_{t}+\Omega \partial_{\phi}, \partial_{\hat{\phi}}=r^{-1} \partial_{\phi}, \nabla \cdot \boldsymbol{u}^{\prime}=\partial_{r} u_{r}^{\prime}+u_{r}^{\prime} / r+$ $\partial_{\hat{\phi}} u_{\phi}^{\prime}+\partial_{z} u_{z}^{\prime}$, and the thermal relaxation term was linearized

$$
\frac{\delta T}{T}=\frac{\delta p}{p}-\frac{\delta \rho}{\rho},
$$

Table 1

Symbols Used in This Work

as per the equation of state, $p=c_{v}(\gamma-1) \rho T$. Next we use the short-wave approximation, $m \ll k_{r} r, k_{z} z$, and expand the perturbations in Fourier modes, $\exp \left(-i \omega t+i k_{r} r+i m \phi+i k_{z} z\right)$. Equations (4)-(8) then become

$$
\begin{aligned}
-i \bar{\omega} \rho^{\prime}+u_{r}^{\prime} \partial_{r} \rho_{0}+\rho_{0} i k_{r} u_{r}^{\prime}+\rho_{0} i k_{z} u_{z}^{\prime} & =0 \\
-i \bar{\omega} u_{r}^{\prime}-2 \Omega u_{\phi}^{\prime}+i k_{r} \rho_{0}^{-1} p^{\prime}-\frac{\rho^{\prime}}{\rho_{0}^{2}} \partial_{r} p_{0} & =0 \\
-i \bar{\omega} u_{\phi}^{\prime}+\Omega(2-q) u_{r}^{\prime} & =0 \\
-i \bar{\omega} u_{z}^{\prime}+i k_{z} \rho_{0}^{-1} p^{\prime} & =0 \\
-i \bar{\omega} p^{\prime}+u_{r}^{\prime} \partial_{r} p_{0}+\rho_{0} c^{2} i k_{r} u_{r}^{\prime}+\rho_{0} c^{2} i k_{z} u_{z}^{\prime} & \\
+\frac{p^{\prime}}{\tau}-\frac{c^{2} \rho^{\prime}}{\gamma \tau} & =0
\end{aligned}
$$


where $\bar{\omega}=\omega-m \Omega$, and we also substituted $p_{0}=\rho_{0} c^{2} / \gamma$. The system is $\mathbf{M} \cdot \boldsymbol{v}=0$, where $\boldsymbol{v}=\left[\rho^{\prime}, u_{r}^{\prime}, u_{\phi}^{\prime}, u_{z}^{\prime}, p^{\prime}\right]^{T}$, and the coefficient matrix is

$$
\begin{aligned}
& \mathbf{M}= \\
& {\left[\begin{array}{ccccc}
-i \bar{\omega} & \rho_{0}\left(i k_{r}+A / c\right) & 0 & \rho_{0} i k_{z} & 0 \\
-B c / \rho_{0} & -i \bar{\omega} & -2 \Omega & 0 & i k_{r} / \rho_{0} \\
0 & \Omega(2-q) & -i \bar{\omega} & 0 & 0 \\
0 & 0 & 0 & -i \bar{\omega} & i k_{z} / \rho_{0} \\
-c^{2} / \gamma \tau & \rho_{0} c^{2}\left(i k_{r}+B / c\right) & 0 & \rho_{0} c^{2} i k_{z} & -i \bar{\omega}+1 / \tau
\end{array}\right]}
\end{aligned}
$$

We substituted

$$
\begin{gathered}
A=c \partial_{r} \ln \rho, \\
B=\gamma^{-1} c \partial_{r} \ln p,
\end{gathered}
$$

so both $A$ and $B$ have dimensions of frequency. In particular, $A B=1 / \rho^{2} \partial_{r} \rho \partial_{r} p$, and $B^{2}=(\gamma \rho p)^{-1}\left(\partial_{r} p\right)^{2}$, so $N^{2}=$ $A B-B^{2}$ is the square of the Brunt-Väisälä frequency. The full dispersion relation $\operatorname{det} \mathbf{M}=0$ is

$$
\begin{aligned}
\bar{\omega}^{5} & +\bar{\omega}^{4} i \tau^{-1}-\bar{\omega}^{3}\left(A B+c^{2} k^{2}+\kappa^{2}\right) \\
& +\bar{\omega}^{2} \tau^{-1}\left[k_{r} c\left(B-A \gamma^{-1}\right)-i\left(A B+c^{2} k^{2} \gamma^{-1}+\kappa^{2}\right)\right] \\
& +\bar{\omega} c^{2} k_{z}^{2}\left(\kappa^{2}+N^{2}\right)+\frac{i c^{2} \kappa^{2} k_{z}^{2}}{\gamma \tau}=0
\end{aligned}
$$

where $\kappa^{2}=2(2-q) \Omega^{2}$ is the square of the epicyclic frequency. We consider now some limits of Equation (17).

\section{ANELASTIC LIMIT}

In the anelastic limit, $c=\infty$, Equation (17) reduces to

$$
\begin{aligned}
& \bar{\omega}^{3} k^{2}-\bar{\omega}^{2} \tau^{-1}\left[k_{r}(b-a / \gamma)-i k^{2} \gamma^{-1}\right] \\
&-\bar{\omega} k_{z}^{2}\left(\kappa^{2}+N^{2}\right)-\frac{i \kappa^{2} k_{z}^{2}}{\gamma \tau}=0,
\end{aligned}
$$

where $b=B / c=\gamma^{-1} \partial_{r} \ln p$ and $a=A / c=\partial_{r} \ln \rho$. These terms are proportional to $1 / r$, so they are small and can be dropped. The dispersion relation is thus

$$
\bar{\omega}^{3}+i \varsigma \bar{\omega}^{2}-\bar{\omega} \mu^{2}\left(\kappa^{2}+N^{2}\right)-i \varsigma \kappa^{2} \mu^{2}=0,
$$

where we have also substituted $\varsigma=1 / \gamma \tau$ and $\mu^{2}=k_{z}^{2} / k^{2}$.

\subsection{Adiabatic}

For adiabatic flow, $\tau=\infty$, Equation (19) reduces to

$$
\bar{\omega}^{2}=\mu^{2}\left(\kappa^{2}+N^{2}\right) .
$$

For $k_{r}=0$ (in-plane incompressible motion), we retrieve $\bar{\omega}^{2}=\kappa^{2}+N^{2}$, the Solberg-Hoiland criterion.

$$
\text { 3.2. Finite } \tau, k_{r}=0
$$

For pure in-plane incompressible motions $\left(k_{r}=0\right)$, Equation (19) reduces to

$$
\bar{\omega}^{3}+\bar{\omega}^{2} i \varsigma-\bar{\omega}\left(\kappa^{2}+N^{2}\right)-i \varsigma \kappa^{2}=0,
$$

which is the same as derived by KH14 (their Equation (18)), using other assumptions.

\subsection{Finite $\tau, k_{r} \neq 0$}

Substituting $\bar{\omega}=s+i \sigma$, growing solutions correspond to real positive $\sigma$. The dispersion relation, real and imaginary, that need to vanish independently, are

$$
\begin{gathered}
s^{2}=\mu^{2}\left(N^{2}+\kappa^{2}\right)+3 \sigma^{2}+2 \sigma \varsigma ; \\
\sigma^{3}+\sigma^{2} \varsigma-\sigma\left[3 s^{2}-\mu^{2}\left(N^{2}+\kappa^{2}\right)\right]-\varsigma\left(s^{2}-\mu^{2} \kappa^{2}\right)=0 .
\end{gathered}
$$

Substituting Equation (22) into Equation (23), we get

$$
2 \sigma(2 \sigma+\varsigma)^{2}+2 \sigma \mu^{2}\left(\kappa^{2}+N^{2}\right)+\mu^{2} \varsigma N^{2}=0 .
$$

As we expect the growth to be small (to be checked a posteriori), we take the limit $\sigma \ll \varsigma$, leading to

$$
\sigma=-\frac{1}{2}\left[\frac{\mu^{2} \varsigma N^{2}}{\varsigma^{2}+\mu^{2}\left(\kappa^{2}+N^{2}\right)}\right] .
$$

This function has no extrema for finite $\mu$. For $\varsigma$, however, there is a maximum at $\left.\varsigma^{2}\right|_{d_{t} \sigma=0}=\varsigma_{\max }^{2}=\mu^{2}\left(\kappa^{2}+N^{2}\right)$, that is, maximum growth occurs for

$$
\tau_{\max }=\frac{1}{\gamma}\left|\frac{k}{k_{z}}\right| \frac{1}{\sqrt{\kappa^{2}+N^{2}}}
$$

for which the growth rate is $\sigma_{\max }=-\mu^{2} N^{2} /\left(4 \zeta_{\max }\right)$, i.e.,

$$
\sigma_{\max }=-\frac{1}{4}\left|\frac{k_{z}}{k}\right| \frac{N^{2}}{\sqrt{\kappa^{2}+N^{2}}} .
$$

\subsubsection{Keplerian Disks}

Recalling the definition of the Brunt-Väisälä frequency

$$
N^{2} \equiv \frac{1}{\rho} \frac{d p}{d r}\left(\frac{1}{\rho} \frac{d \rho}{d r}-\frac{1}{\gamma p} \frac{d p}{d r}\right),
$$

we can write it in terms of the power-law indices of the density and temperature gradients, $\alpha=\partial \ln \rho / \partial \ln r$, and $\xi=\alpha+\beta=$ $\partial \ln P / \partial \ln r$, resulting in

$$
N^{2}=\frac{\Omega^{2} h^{2}}{\gamma}\left(\alpha \xi-\frac{1}{\gamma} \xi^{2}\right),
$$

where $h=H / r$ is the aspect ratio and $H=c / \Omega$ the scale height. So, for Keplerian disks, $\kappa=\Omega$ and $\left|N^{2}\right| \sim \Omega^{2} \mathcal{O}\left(h^{2}\right)$. From this, $\tau_{\max }$ is of order $1 / \Omega$, while the associated growth rate is of order $\sigma_{\max }=\Omega \mathcal{O}\left(h^{2}\right)$, validating the assumption that $\sigma \ll \varsigma$.

Notice that for $k_{r} \gg k_{z}$, that is $\mu^{2} \rightarrow 0$, the dispersion relation (Equation (24)) becomes

$$
\sigma(2 \sigma+\varsigma)^{2}=0,
$$

for which the roots are $\sigma=0$, and $\sigma=-\varsigma / 2$, that is, no growth and damped perturbations. For channel modes $\left(k_{r}=0\right)$ in Keplerian disks $(\kappa=\Omega \gg|N|)$, we find

$$
\begin{gathered}
\tau_{\max }=\frac{1}{\gamma \Omega} ; \\
\sigma_{\max }=-\frac{N^{2}}{4 \Omega} .
\end{gathered}
$$




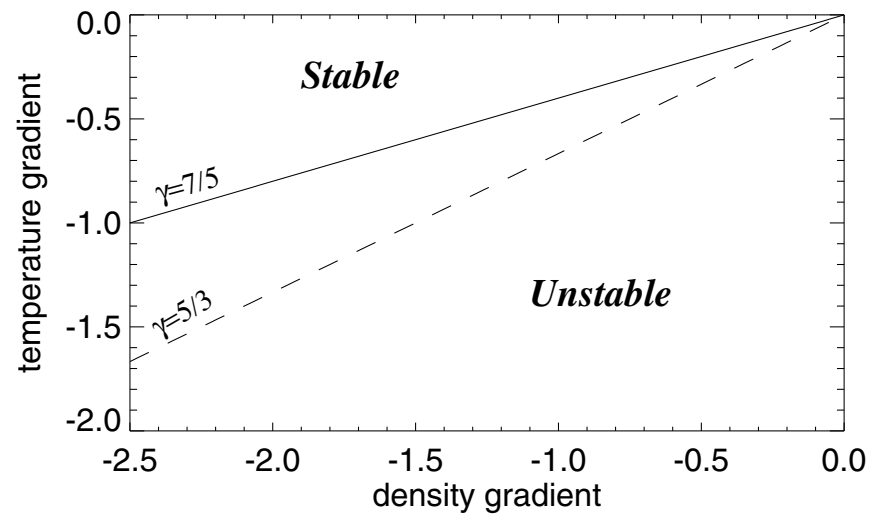

Figure 1. Sign of the square of the Brunt-Väisälä frequency defines the stability criterion, shown here as a function of the density and temperature power-law indices. The plot shows the lines for two values of $\gamma$. Above (below) the respective line the system is stable (unstable).

We plot in Figure 1 the unstable range as a function of the density and temperature power-law indices. ${ }^{4}$

\subsection{The Unstable Mode}

To understand the most unstable mode, we check the eigenvector $\boldsymbol{v}_{\max }$ corresponding to this root, for which the eigenvalue is

$$
\lambda=i \omega_{\max }=i \Omega-\sigma_{\max },
$$

and the system is $\mathbf{R} \cdot \boldsymbol{v}_{\max }=\lambda \boldsymbol{v}_{\max }$, where

$$
\mathbf{R}=\left[\begin{array}{ccccc}
0 & \rho_{0} A / c & 0 & \rho_{0} i k_{z} & 0 \\
-B c / \rho_{0} & 0 & -2 \Omega & 0 & 0 \\
0 & \Omega / 2 & 0 & 0 & 0 \\
0 & 0 & 0 & 0 & i k_{z} / \rho_{0} \\
-c^{2} \Omega & \rho_{0} B c & 0 & \rho_{0} c^{2} i k_{z} & \gamma \Omega
\end{array}\right]
$$

The fourth line is $i k_{z} / \rho_{0} u_{z}^{\prime}=i \Omega u_{z}^{\prime}-\sigma_{\max } u_{z}^{\prime}$, which is only satisfied for the trivial solution $u_{z}^{\prime}=0$. The reduced system becomes

$$
\begin{aligned}
& \rho^{\prime}=\lambda^{-1} \rho_{0} a u_{r}^{\prime} ; \\
& u_{\phi}^{\prime}=\lambda^{-1} \Omega / 2 u_{r}^{\prime} ; \\
& p^{\prime}=(\lambda-\gamma \Omega)^{-1}\left(\rho_{0} c B u_{r}^{\prime}-c^{2} \Omega \rho^{\prime}\right) .
\end{aligned}
$$

The solution is

$$
\begin{aligned}
\ln \rho^{\prime} & =-\frac{\sigma+i \Omega}{\Omega} a u_{r}^{\prime} \\
u_{\phi}^{\prime} & =-\frac{\sigma+i \Omega}{2 \Omega} u_{r}^{\prime}
\end{aligned}
$$

4 Note that the condition that $N^{2}<0$ requires (for $\xi<0$ ) that $\alpha-\frac{\beta}{(\gamma-1)}>0$. For a power-law surface density $\Sigma \propto \rho H \propto r^{\psi}$, we have $\psi=\alpha+\frac{\beta}{2}+\frac{3}{2}$. The requirement is then $2 \psi>3+\beta \frac{(\gamma+1)}{(\gamma-1)}$, which for $\gamma=\frac{7}{5}$ means $\psi>3\left(\beta+\frac{1}{2}\right)$. For $\beta=-\frac{1}{2}$, the surface density has to be flat or increasing with distance in order to lead to instability, which is not reasonable. For $\beta=-\frac{3}{4}$, the onset of instability corresponds to $\psi=-\frac{3}{4}$ (also for $\gamma=\frac{7}{5}$ ), which is consistent with the range of $\psi \approx[-0.4,-1.0]$ (with median -0.9 ) found in the observations of Andrews et al. (2009).

$$
\ln p^{\prime}=-\gamma\left(\frac{\sigma}{\Omega} a+b\right)\left[\frac{\sigma+\Omega \gamma+i \Omega}{(\sigma+\gamma \Omega)^{2}+\Omega^{2}}\right] u_{r}^{\prime} .
$$

Since $\sigma \ll \Omega$, the pressure perturbation is

$$
\ln p^{\prime}=-\frac{\gamma(\gamma+i)}{\Omega(\gamma+1)} b u_{r}^{\prime} .
$$

And, because $a$ and $b$ are of order $1 / r, \ln \rho^{\prime}$ and $\ln p^{\prime}$ are vanishingly small. That the pressure variation does not play a major role in the instability justifies (now a posteriori) the $p^{\prime}=0$ approximation of KH14. The eigenvector is simply

$$
\boldsymbol{v}_{\max }=\left[0,1,-\frac{1}{2}\left(\frac{\sigma}{\Omega}+i\right), 0,0\right]^{T}
$$

i.e., an overstable epicycle.

\section{NUMERICAL SIMULATIONS}

We now turn to numerical simulations to check the evolution of the instability. We use the shearing box model of Lyra \& Klahr (2011), which includes the linearized pressure gradient. We do so in order to benefit from shear-periodic boundaries, in contrast to the simulations in the Appendix of KH14, which are affected by radial boundaries. The reader is referred to Lyra $\&$ Klahr (2011) for the equations of motion and properties and caveats of the approximation. In particular, the density gradient is zero, and we drop the $x$-dependent term in the $p d V$ work to keep shear-periodicity (see Appendix A of Lyra \& Klahr 2011).

We solve the evolution equations with the PENCIL CODE (Brandenburg \& Dobler 2002) $)^{5}$ which integrates the partial differential equations with sixth-order spatial derivatives, and a third-order Runge-Kutta time integrator. Sixth-order hyperdissipation terms are added to the evolution equations, to provide extra dissipation near the grid scale, explained in Lyra et al. (2008a). They are needed because the high-order scheme of the PENCIL CODE has little overall numerical dissipation (McNally et al. 2012).

We run a suite of two-dimensional axisymmetric models ( $x$ and $z$ ) to understand the linear evolution and saturation properties of the instability. The sound speed is $c=0.1$, and the adiabatic index is $\gamma=1.4$. The cooling time is $\tau_{\max }=1 / \gamma \Omega$. Our units are $\Omega=c_{p}=\rho_{0}=1$.

We initialize the simulations with the eigenvector corresponding to the epicycle oscillation, $u_{y}=-i u_{x} / 2$. Because for $k_{x}=0$ the growth rate does not depend on $k_{z}$, we arbitrarily choose $\lambda_{z}=H$ for the channel mode. The initial condition therefore is

$$
\begin{aligned}
& u_{x}=u_{0} \sin \left(\frac{2 \pi}{H} z\right) \\
& u_{y}=\frac{u_{0}}{2} \cos \left(\frac{2 \pi}{H} z\right) .
\end{aligned}
$$

The fiducial model has resolution $\Delta x=\Delta z=H / 64$, box size $L_{x} \times L_{z}=2 H \times 2 H$, temperature gradient $\beta=-3.5$, and initial amplitude $u_{0} / c=10^{-3}$. We vary these quantities to check convergence at saturation. The evolution of the twodimensional axisymmetric box seeded with the channel mode

\footnotetext{
5 The code, including improvements done for the present work, is publicly available under a GNU open source license and can be downloaded at http://www.nordita.org/software/pencil-code.
} 
(a)

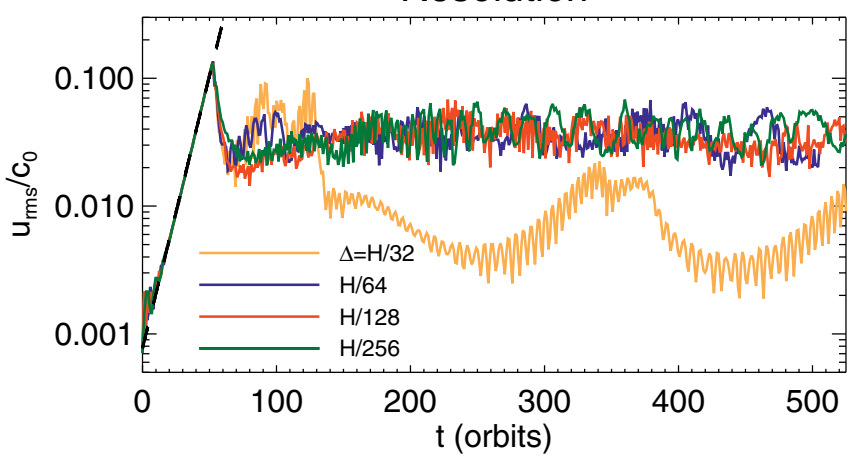

(c)

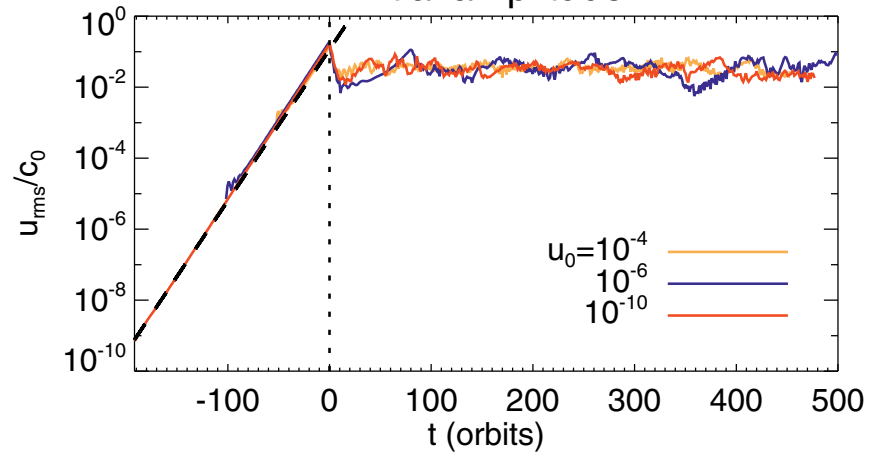

(b)

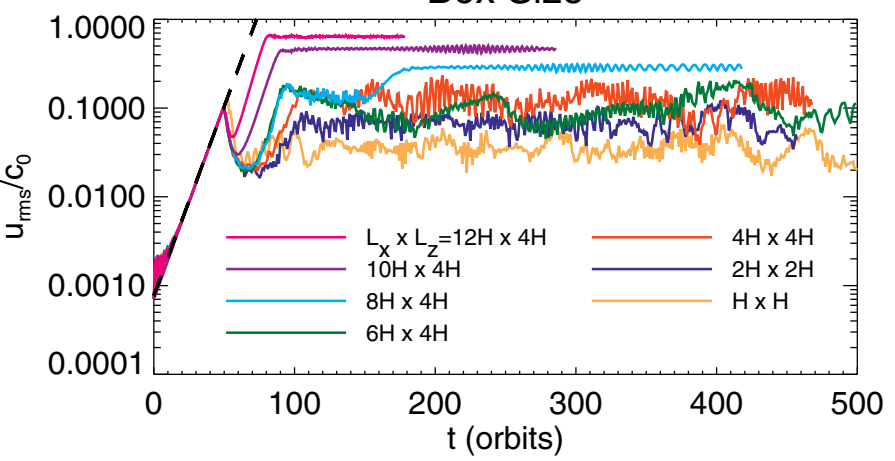

(d)

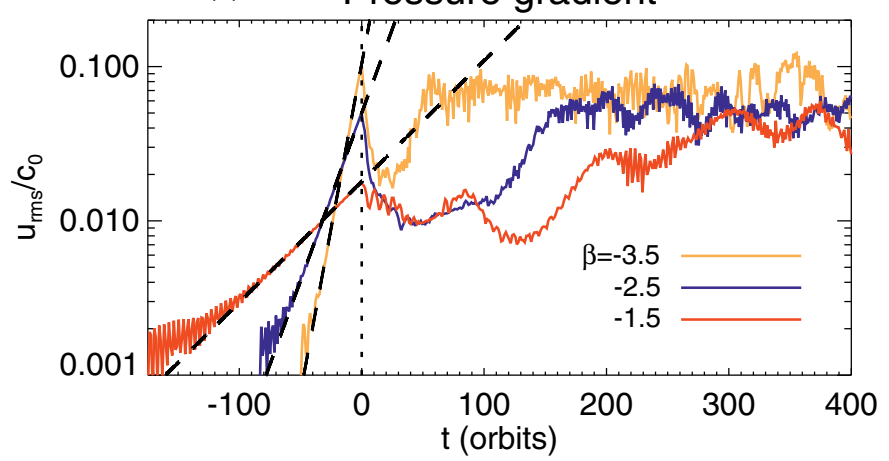

Figure 2. Convergence study of the saturated state of the overstability. (a) The box size $(H \times H)$, initial amplitude $\left(u_{\mathrm{rms}} / c=10^{-3}\right)$, and pressure gradient $(\xi=-3.5)$ were kept fixed, while the resolution was changed. Saturation occurs at 64 points per scale height. (b) The resolution is fixed at $\Delta=H / 64$, and the box size changed. There is no convergence with box size (see Figure 3 and the discussion in the text). (c) Resolution $\Delta=H / 64$, box size $H \times H$, and varying initial amplitude. (d) Varying the pressure gradient, resolution $\Delta=H / 64$, box size $2 H$. Amplitude converges in the latter two cases. The linear growth rate (black dashed line) is very well reproduced in all cases.

(A color version of this figure is available in the online journal.)
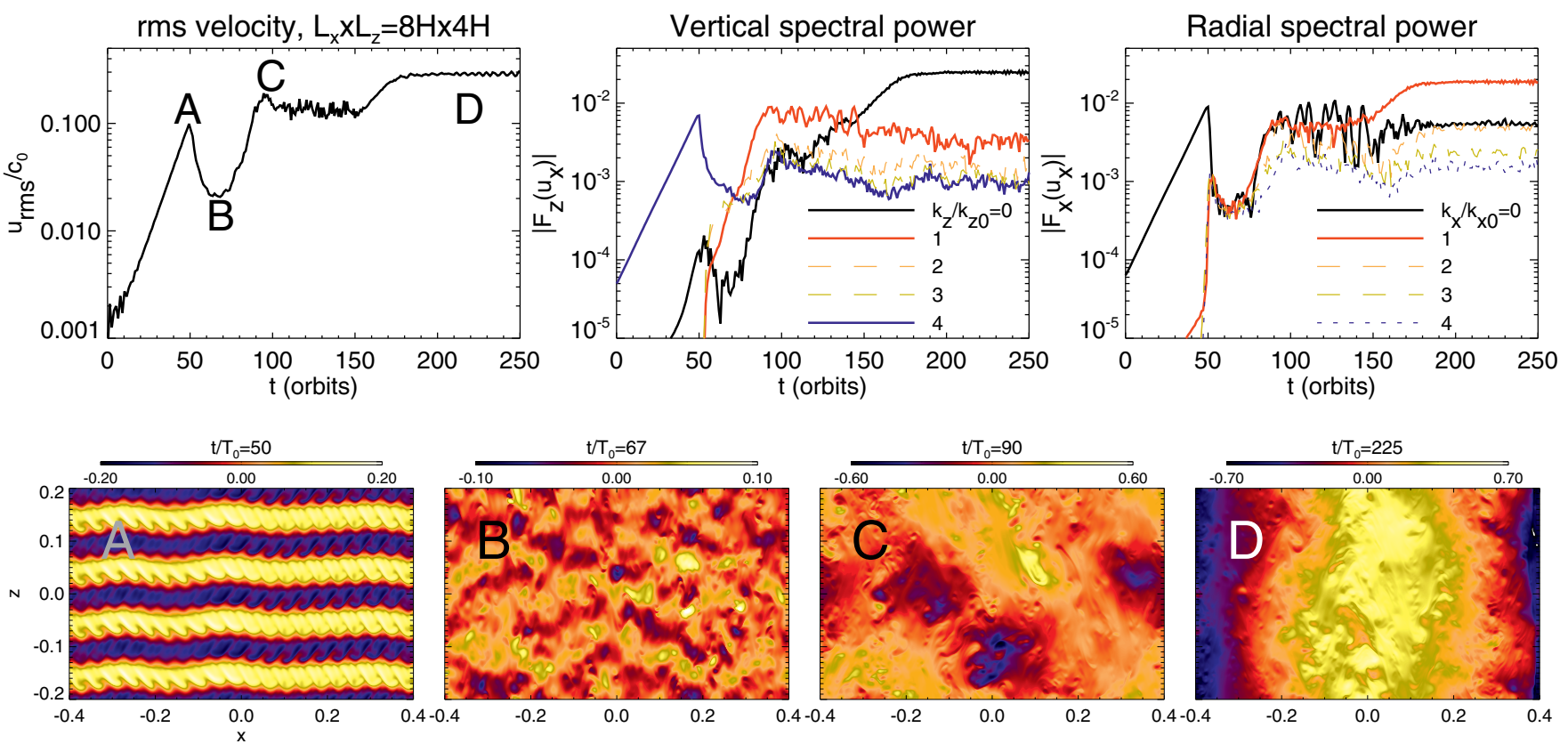

Figure 3. Spectral description of the $8 H \times 4 H$ simulation (cyan line in Figure 2, upper right). The rms velocity is shown in the upper left panel, with four representative points marked, and the velocity shown, in the $x z$ plane, in each of these points (lower panels). The points are $A$, onset of saturation; $B$, the local minimum; $C$, second saturated state; and $D$, state after the last bifurcation. The spectral power in the first five large-scale modes in $x$ and $z$ is shown in the upper middle and upper right panels, respectively. Point $A$ corresponds to Kelvin-Helmholtz instability breaking up the original $k_{z} / k_{z 0}=4$ channel mode, as nonzero $k_{x}$ and $k_{z}$ modes are excited. The local minimum $B$ corresponds to the point when power is equally distributed among the non-zero $k_{z}$ modes. The saturated state $C$ corresponds to the dominance of the $k_{z}=1$ mode, with a mixed $k_{x}=1$ mode (both clear in the lower " $\mathrm{C}$ " panel). The last bifurcation corresponds to the $k_{z}=0$ mode taking over and a large scale $k_{x}=1$ dominating the box.

(A color version of this figure is available in the online journal.) 

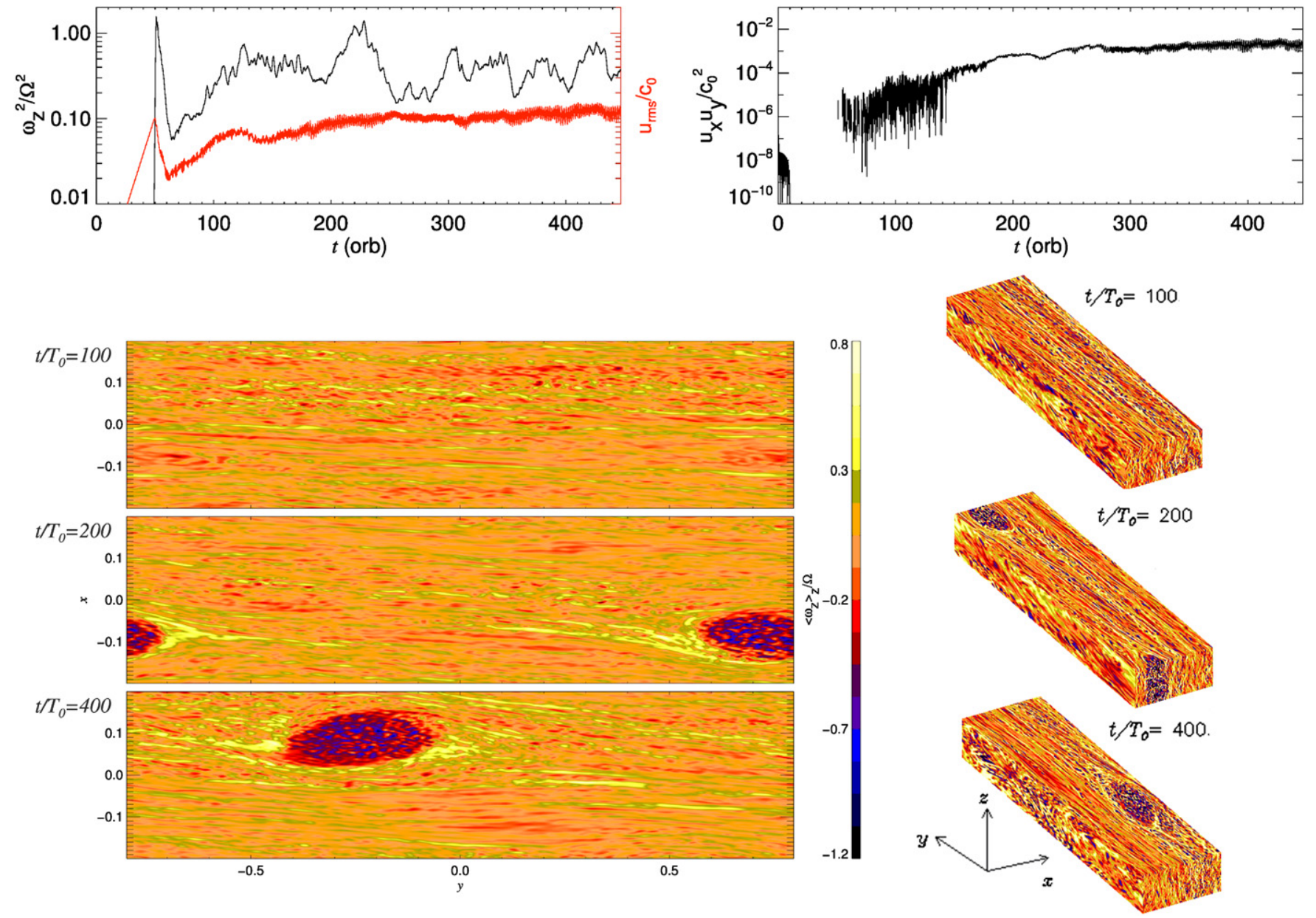

Figure 4. Nonlinear evolution of the buoyant overstability in three dimensions. With the linear overstability raising the amplitude of the initial fluctuations to nonlinear levels, the saturated state is expected to be similar to that of the SBI. In the lower left panels we show the averaged vertical vorticity; the lower right panel shows the vorticity in the three-dimensional flow. Indeed, we see that large-scale self-sustained anticyclonic vortices develop in the saturated state. The upper left panel shows the radial velocity rms and vertical enstrophy (red and black line, respectively.) The upper right panel shows the level of Reynolds stress, which saturates at $\alpha \approx 10^{-3}$.

(A color version of this figure is available in the online journal.)

is shown in the panels of Figure 2. The linear phase matches the analytical prediction (dashed black line) for all models ran.

Figure 2(a) shows the dependency on resolution. Convergence is achieved for 64 grid points per scale height. There is also convergence for the initial amplitude of the perturbation, as seen in Figure 2(c). The linear phase is identical in the three cases examined $\left(u_{0}=10^{-4}, 10^{-6}\right.$, and $\left.10^{-10}\right)$. In this figure we set $t=0$ as the time when saturation is achieved, to better compare with the nonlinear evolution. In Figure 2(d) we check how the instability depends on the pressure gradient. Again, the linear phase is reproduced for the different values of the Brunt-Väisälä frequency, and the amplitudes at saturation are similar, within a factor two to three. A difference in seen when we test the dependency on box size (Figure 2(b)). The amplitude seemed to saturate at $4 H \times 4 H$ (red line), since the model with $L_{x}=6 H$ (green line) shows a similar amplitude. However, the model with $L_{x}=8 H$ (cyan line) shows a bifurcation at $\approx 150$ orbits. Models with a larger radial range ( $L_{x}=10$ and $L_{x}=12$, purple and magenta lines, respectively) show no convergence, even as the velocity dispersion increasingly approaches the sound speed.

Interesting features that help us understand the behavior of the system are seen in this simulation. We plot in Figure 3 the time evolution of the power in the first five large-scale modes, in both $x$ (upper middle panel) and $z$ (upper left panel). The upper left panel shows the rms of the radial velocity. Four special/ representative instants are labeled, and the $u_{x}$ field for these respective instants are shown in the lower panels.

The first instant, $A$, corresponds to the first "saturation" seen at 50 orbits. The power spectrum shows that the clean initial channel mode $\left(k_{z} / k_{z 0}=4, k_{x} / k_{x 0}=0\right)$ persisted until this time, after which it saturates, exciting $k_{x} \neq 0$ modes and other $k_{z}$ modes. Instant $B$, at 67 orbits, corresponds to the local minimum in rms velocity. The power spectrum shows that this happens when the $k_{z} / k_{z 0}=1$ mode becomes dominant. Subsequently, this mode keeps growing, at the same rate as the initial $k_{z} / k_{z 0}=4$ mode. This is because the growth rate is independent of $k_{z}$ for $k_{x}=0$, which at that time has similar power to the higher $k_{x}$ modes. From time $t=90$ (instant $C$ ) to 160 orbits the system settles into a steady state, with a dominant $k_{z} / k_{z 0}=1$ mode, and mixed $k_{x} / k_{x 0}=0$ and $k_{x} / k_{x 0}=1$. Another bifurcation happens when the $k_{z}=0$ mode overtakes the $k_{z} / k_{z 0}=1$ mode. Simultaneously, it prompts $k_{x} / k_{x 0}=1$ to dominate over $k_{x}=0$. The final state (labeled $D$ ) is thus vertically symmetric, with a box-wide radial wavelength.

This explains why we do not find convergence while increasing the box vertical range from $L_{z}=H$ to $2 H$ to $4 H$. In these boxes, because we kept the seed mode at $k_{z}=2 \pi / H$, we initialized the instability with the $k_{z} / k_{z 0}=1,2$, and 4 modes, 
respectively. In the last two simulations, the $k_{z} / k_{z 0}=1$ mode was growing, with less power, but eventually catching up as the seed mode saturates. Convergence with radial box size is never achieved in the two-dimensional runs because the $k_{x} / k_{x 0}=1$ mode comes to dominate, no matter how wide we make the box. The simulations with radial box size $L_{x}=10 H$ and $L_{x}=12 H$ show the same pattern, albeit with no intermediate phase of dominance of a $k_{z} / k_{z 0}=1$ mode.

\subsection{Three-dimensional Instability: Growth of Large-scale Vortices}

Next we turn to the three-dimensional evolution of the instability. We set a box of size $4 H \times 16 H \times 2 H$, with resolution $256 \times 256 \times 128$ in $x, y$, and $z$, respectively. The cells thus have aspect ratio $1 \times 4 \times 1$ (we have checked in Lyra \& Klahr 2011 that the unit aspect ratio in $x$ and $y$ gave the same results for the two-dimensional SBI).

With the azimuthal direction present, vertical vorticity (inplane circulation) can evolve unabridged. We show in Figure 4 (left panel) the evolution of the rms velocity (red line) and enstrophy (black line). When the initial $k_{z}$ mode saturates (at 50 orbits, as in the two-dimensional meridional models of Figure 3), a sharp rise in enstrophy occurs. The situation is now very similar to the SBI, with high-amplitude perturbations $\left(u_{\mathrm{rms}} \approx 0.1 c_{s}\right)$, thermal relaxation, and an entropy gradient. The nonlinear saturation state of this buoyant overstability should thus proceed very similarly to the evolution of the SBI. Indeed, as the lower panels of Figure 4 show, the saturated state develops into a large-scale vortex. The amount of angular momentum transport (Figure 4, upper right) is at the $\alpha \approx 10^{-3}$ level, again, the typical level of the SBI. It seems conclusive that the saturated state of the buoyant overstability is the SBI.

\section{CONCLUSIONS}

We conclude that indeed there is a linear overstability in the region of the parameter space of negative $N^{2}$, finite cooling time $\tau$, and non-zero $k_{z}$ perturbation. The approximation $\delta p=0$ done by KH14 is justified since $\delta p=0$ (and $\delta \rho$ ) in the eigenvector of the most unstable modes is vanishingly small in comparison to the velocity amplitude (Equation (42)).

Modeling the system numerically, we reproduce the linear growth rate in all cases. In the two-dimensional meridional simulations, we find convergence in the saturated state with resolution, but not with box size, since a large-scale $k_{x} / k_{x 0}=1$ radial mode dominates the box. However, in three dimensions this mode does not show up, as it gets sheared away.

We also show that the SBI is indeed the saturated state of the overstability. Saturation leads to a fast burst of enstrophy in the box, and a large-scale vortex develops in the course of the next $\approx 100$ orbits after the convective overstability has built the finite amplitude perturbations. The amount of angular momentum transport achieved is of the order of $\alpha \approx 10^{-3}$, as in compressible SBI models.

It remains to be shown whether these processes (both SBI and convective overstability) operate in global models, i.e., how they respond to boundary conditions and curvature terms. The relation between this overstability and the Goldreich-Schubert-Fricke instability (Goldreich et al. 1967; Fricke 1968; Nelson et al. 2013) should also be the subject of future work.

This work was performed in part at the Jet Propulsion Laboratory, under contract with the California Institute of Technology funded by the National Aeronautics and Space Administration (NASA) through the Sagan Fellowship Program executed by the NASA Exoplanet Science Institute. This paper started from a discussion between the author, Alexander Hubbard, Matthew Kunz, Hubert Klahr, Henrik Latter, Geoffroy Lesur, Min-Kai Lin, George Mamatsashvili, and Orkan Umurhan. It further profited from input from Anders Johansen, Mordecai-Mark Mac Low, Colin McNally, Neal Turner, and Andrew Youdin.

\section{REFERENCES}

Andrews, S. M., Wilner, D. J., Hughes, A. M., Qi, C., \& Dullemond, C. P. 2009, ApJ, 700, 1502

Balbus, S. A., \& Hawley, J. F. 1991, ApJ, 376, 214

Barge, P., \& Sommeria, J. 1995, A\&A, 295, L1

Blaes, O. M., \& Balbus, S. A. 1994, ApJ, 421, 163

Brandenburg, A., \& Dobler, W. 2002, CoPhC, 147, 471

Fricke, K. 1968, ZA, 68, 317

Gammie, C. F. 1996, ApJ, 457, 355

Goldreich, P., \& Schubert, G. 1967, ApJ, 150, 571

Heinemann, T., \& Papaloizou, J. C. B. 2009a, MNRAS, 397, 52

Heinemann, T., \& Papaloizou, J. C. B. 2009b, MNRAS, 397, 64

Heinemann, T., \& Papaloizou, J. C. B. 2012, MNRAS, 419, 1085

Johnson, B. M., \& Gammie, C. F. 2005, ApJ, 635, 149

Klahr, H. 2004, ApJ, 606, 1070

Klahr, H., \& Bodenheimer, P. 2003, ApJ, 582, 869

Klahr, H., \& Bodenheimer, P. 2006, ApJ, 639, 432

Klahr, H., \& Hubbard, A. 2014, ApJ, 788, 21

Lesur, G., \& Papaloizou, J. C. B. 2010, A\&A, 513, 60

Lyra, W., Johansen, A., Klahr, H., \& Piskunov, N. 2008a, A\&A, 479, 883

Lyra, W., Johansen, A., Klahr, H., \& Piskunov, N. 2008b, A\&A, 491, L41

Lyra, W., Johansen, A., Zsom, A., Klahr, H., \& Piskunov, N. 2009, A\&A, 497, 869

Lyra, W., \& Klahr, H. 2011, A\&A, 527A, 138

McNally, M., Lyra, W., \& Passy, J.-C. 2012, ApJS, 201, 18

Nelson, R. P., Gressel, O., \& Umurhan, O. M. 2013, MNRAS, 435, 2610

Petersen, M. R., Julien, K., \& Stewart, G. R. 2007a, ApJ, 658, 1236

Petersen, M. R., Stewart, G. R., \& Julien, K. 2007b, ApJ, 658, 1252

Raettig, N., Lyra, W., \& Klahr, H. 2013, ApJ, 765, 115

Shakura, N. I., \& Sunyaev, R. A. 1973, A\&A, 24, 337

Turner, N. J., \& Drake, J. F. 2009, ApJ, 703, 2152 